\title{
Corrigendum
}

\section{Growth of total fat and lean and of primal cuts is affected by the sex type - CORRIGENDUM}

\author{
A. Carabús, R. D. Sainz, J. W. Oltjen, M. Gispert and M. Font-i-Furnols \\ doi: 10.1017/S1751731117000039, published by Cambridge University Press, 10 February 2017.
}

In Table 1 the incorrect bone volume by sex was presented. The correct table is shown below. The authors apologise for the error.

Table 1 Volumes of lean, fat and bone and target body weight $(\mathrm{TBW})^{*}$ of castrated male (CM), female (FE), immunocastrated male (IM), and entire male (EM) pigs

\begin{tabular}{|c|c|c|c|c|c|c|c|c|}
\hline \multirow[b]{2}{*}{ Volume $\left(\mathrm{dm}^{3}\right)$} & \multirow[b]{2}{*}{ TBW } & \multirow[b]{2}{*}{$\mathrm{CM}$} & \multirow[b]{2}{*}{$\mathrm{FE}$} & \multirow[b]{2}{*}{ IM } & \multirow[b]{2}{*}{ EM } & \multicolumn{3}{|c|}{$P$-value } \\
\hline & & & & & & SEX & TBW & SEX*TBW \\
\hline \multirow[t]{5}{*}{ Lean } & 30 & 21.48 & 20.92 & 21.66 & 22.6 & & & \\
\hline & 70 & $43.503^{b}$ & $44.72^{a b}$ & $47.66^{\mathrm{a}}$ & $48.13^{a}$ & & & \\
\hline & 100 & $56.93^{c}$ & $62.25^{\mathrm{b}}$ & $62.43^{\mathrm{ab}}$ & $65.71^{a}$ & & & \\
\hline & 120 & $65.73^{c}$ & $71.41^{b}$ & $69.43^{b}$ & $75.98^{\mathrm{a}}$ & & & \\
\hline & SE & 0.68 & 0.68 & 0.68 & 0.71 & $<0.0001$ & $<0.0001$ & $<0.0001$ \\
\hline \multirow[t]{5}{*}{ Fat } & 30 & 4.68 & 4.74 & 3.52 & 3.69 & & & \\
\hline & 70 & $16.52^{\mathrm{a}}$ & $14.42^{\mathrm{ab}}$ & $11.75^{b}$ & $12.49^{\mathrm{ab}}$ & & & \\
\hline & 100 & $28.96^{\mathrm{a}}$ & $24.81^{a b}$ & $23.65^{b c}$ & $20.44^{c}$ & & & \\
\hline & 120 & $38.31^{a}$ & $31.89^{\mathrm{b}}$ & $33.38^{b}$ & $25.39^{c}$ & & & \\
\hline & SE & 0.86 & 0.86 & 0.86 & 0.90 & $<0.0001$ & $<0.0001$ & $<0.0001$ \\
\hline \multirow[t]{5}{*}{ Bone } & 30 & 2.36 & 2.28 & 2.43 & 2.49 & & & \\
\hline & 70 & 4.55 & 4.55 & 5.00 & 4.91 & & & \\
\hline & 100 & 5.85 & 6.07 & 6.30 & 6.44 & & & \\
\hline & 120 & 6.72 & 6.84 & 7.00 & 7.32 & & & \\
\hline & SE & 0.12 & 0.12 & 0.12 & 0.12 & $<0.0001$ & $<0.0001$ & 0.467 \\
\hline
\end{tabular}

*Different subscripts within a row indicate significant differences $(P<0.05)$; Lean $=$ Volume of lean $\left(\mathrm{dm}^{3}\right)$ calculated using HU distribution between 1 and 140 Fat $=$ Volume of fat $\left(\mathrm{dm}^{3}\right)$ calculated using HU distribution between -149 and 0 , Bone $=$ Volume of bone $\left(\mathrm{dm}^{3}\right)$ calculated using HU distribution between 141 and 1400

\section{Reference}

Carabús A, Sainz RD, Oltjen JW, Gispert M and Font-i-Furnols M 2017. Growth of total fat and lean and of primal cuts is affected by the sex type. Animal 11, 1321-1329. 